\title{
Appartenances linguistiques, identités collectives et pratiques culturelles en Pays Basque
}

Retour sur une enquête

Linguistic behaviour, collective identities and cultural behaviour in the Basque

Country: revisiting a survey

\section{Xabier Itçaina}

\section{(2) OpenEdition}

Journals

\section{Édition électronique}

URL : http://journals.openedition.org/conflits/18001

DOI : $10.4000 /$ conflits. 18001

ISSN : $1777-5345$

Éditeur :

CCLS - Centre d'études sur les conflits lilberté et sécurité, L'Harmattan

\section{Édition imprimée}

Date de publication : 26 décembre 2010

Pagination : 19-36

ISBN : 978-2-296-56047-5

ISSN : 1157-996X

Référence électronique

Xabier Itçaina, « Appartenances linguistiques, identités collectives et pratiques culturelles en Pays Basque », Cultures \& Conflits [En ligne], 79-80 | Automne/Hiver 2010, mis en ligne le 26 mai 2012,

consulté le 30 mars 2021. URL : http://journals.openedition.org/conflits/18001 ; DOI : https://doi.org/ $10.4000 /$ conflits. 18001 


\title{
Appartenances linguistiques, identités collectives et pratiques culturelles en Pays Basque
}

\author{
Retour sur une enquête
}

\section{Xabier ITÇAINA}

Xabier Itçaina est chargé de recherche CNRS an Centre Émile Durkheim (UMR 5116 du CNRS) à Sciences Po Bordeaux, Université de Bordeaux. Il poursuit ses recherches dans trois directions: les interactions entre religion et politique en Europe du Sud, les dynamiques territoriales de l'économie sociale et solidaire, et l'anthropologie politique des mobilisations territoriales en Pays Basque.

$\mathrm{L}$ ongtemps ${ }^{1}$ réservée aux seuls sociolinguistes, la question des langues est désormais de plus en plus investie par les politistes, en particulier sous l'angle de l'action publique ${ }^{2}$. Prenant acte de ce regain d'intérêt, cet article s'assigne un objectif à la fois empirique et méthodologique en proposant un retour critique sur une enquête croisant enjeux linguistiques, culturels et identitaires. Menée en 2004-2005 en Pays Basque français, Communauté autonome basque (CAB dans le reste du texte) et Navarre, cette enquête a porté sur le thème générique Identité et culture basque au début $d u$ XXIe siècle et a fait l'objet d'une triple édition en espagnol, français et basque ${ }^{3}$. J'ai participé à cette enquête au titre de son comité scientifique, qui associait le bureau de prospective sociologique du gouvernement basque de la CAB, la Société d'études basques Eusko Ikaskuntza et, côté français, l'Institut culturel basque

1. Une première version de ce texte a été discutée lors du Congrès AFSP 2009 à Grenoble. Je remercie les participants à l'atelier et les évaluateurs de la revue pour leurs commentaires, ainsi que l'équipe de coordination de l'enquête de 2004-2005 pour la richesse de nos échanges.

2. Rochas A., «A l'épreuve de la diversité linguistique : sociogenèse de la politique des langues régionales (1992-2008) ", in Martin D.-C. (ed), L'identité en jeux. Pouvoirs, identifications, mobilisations, Paris, Karthala, 2010, pp. 361-377 ; Cole A., Harguindéguy J.-B., « La politique linguistique de la France à l'épreuve des revendications ethnoterritoriales ", Revue française de science politique, vol. 59, $\mathrm{n}^{\circ} 5$, octobre 2009, pp. 939-966.

3. Baxok E., Etxegoin P., Lekumberri T., Martinez de Luna I., Mendizabal L., Ahedo I., Itçaina X., Jimeno R., Euskal nortasuna eta kultura XXI. mendearen hasieran. Identidad y cultura vascas a comienzos del siglo XXI. Identité et culture basques au début du XXIe siècle, SaintSébastien, Eusko Ikaskuntza, 2006. 
(ICB). L'enquête, quantitative et qualitative, a permis de réintégrer la variable linguistique dans un questionnement sur les appartenances identitaires et sur les représentations et pratiques culturelles. Elle a constitué, en ce sens, une innovation par rapport aux enquêtes sociolinguistiques périodiquement répétées des deux côtés de la frontière depuis 1991.

La publication de l'enquête a provoqué des réactions contrastées, du moins en Pays Basque français, sur lequel ce texte concentrera son attention. Ce sont tout autant les conditions de réalisation et les motivations de l'enquête que les résultats proprement dits qui ont soulevé des discussions. Certains acteurs culturels et institutionnels accordent valeur d'expertise à l'enquête dans le processus de construction d'une politique linguistique et culturelle. D’autres, proches des milieux militants, voient dans la démarche une «étude de marché » commanditée par des institutions culturelles en quête de repères. Les conditions de la réception de l'enquête prouvent la teneur politique de toute démarche réflexive sur les liens entre langue, culture et identité, surtout lorsque le volet méthodologique principal, quantitatif, se prête à des interprétations non contextualisées. L'enquête, et l'amorce de débat qu'elle a générée, offrent du même coup une prise nouvelle pour la sociologie politique.

Dans un tel contexte, ce texte souligne que l'enquête de 2005, les conditions de sa production et de sa réception peuvent faire l'objet de deux niveaux de lecture. Le premier est celui des résultats proprement dits, qui viennent confirmer la fragmentation des territoires basques, avec une imbrication différenciée des facteurs identitaires, culturels et linguistiques selon les territoires. Un second niveau de lecture concerne les problèmes méthodologiques auxquels l'équipe de coordination scientifique a été confrontée tout au long de l'enquête : comment mesurer les identités ethnolinguistiques ? Quelle pertinence des approches quantitatives, même compensées par des focus groups ? Comment repérer les dimensions proprement politiques de ces représentations ? Ces données, précisément, ne prennent sens qu'au regard des contrastes des mobilisations collectives et surtout des degrés d'institutionnalisation des politiques linguistiques et culturelles sur les trois territoires. A cet égard, la situation du Pays Basque français est particulièrement parlante. Longtemps perçu comme le pendant sur un mode mineur d'une revendication autrement vigoureuse et institutionnalisée côté espagnol, ce territoire connaît depuis la fin des années 1980 un processus d'institutionnalisation de la défense de la langue et de la culture basque et, plus généralement, du développement territorial. Cette dynamique a consisté en partie à tenter de convertir en logiques d'actions publiques sectorielles des dynamiques multiformes essentiellement portées jusque-là par une société civile mobilisée. La question linguistique est passée du statut de problème public à un problème politique ${ }^{4}$, en enrôlant largement acteurs publics, privés ou associatifs. Le processus

4. Au sens de Lascoumes P., Le Galès P., Sociologie de l'action publique, Paris, A. Colin, 2007. 
d'institutionnalisation de la cause linguistique a entraîné, dans un second temps, une différenciation entre politique linguistique et politique culturelle. De par ce double processus d'institutionnalisation publique et de structuration militante, le cas basque se situe à l'intersection de deux des trois trajectoires qu'empruntent, selon J.-B. Harguindéguy et A. Cole, les mobilisations ethnolinguistiques en France 5. De fait, ce n'est qu'à la lumière de ces débats politiques, qui situent la question linguistique à l'intersection des mouvements sociaux et des politiques territoriales, que les données de l'enquête de 20042005 prennent leur sens véritable.

\section{L'enquête : méthodologie et résultats significatifs}

L'enquête, coordonnée par le Service de prospective sociologique du Gouvernement autonome de la $\mathrm{CAB}$, comportait trois volets. L'enquête quantitative a consisté en l'application d'un questionnaire entre septembre et novembre 2004 auprès d'un échantillon conséquent de 3822 personnes âgées de plus de 16 ans, réparties comme suit : 1204 dans la Communauté autonome basque, 1008 en Navarre, 1610 en Pays Basque français. L'échantillon a été constitué de façon représentative (sexe, emploi, âge, résidence) sur chaque territoire ${ }^{6}$. Il est à souligner que l'échantillon basque français, par son ampleur, est particulièrement représentatif, notamment eu égard aux enquêtes équivalentes menées à la fin des années 1970. Si certains items ont dû être adaptés à chaque territoire, la grande majorité du protocole a été appliquée de façon rigoureusement similaire auprès de l'ensemble des enquêtés, ce qui a autorisé une exploitation comparative des résultats. Un deuxième volet de l'enquête, qualitatif, a réuni des groupes de discussion entre octobre 2004 et juillet 2005. 22 groupes ont été constitués, 7 entretiens individuels et 3 récits de vie ont été recueillis. Enfin, le troisième volet de l'enquête a porté sur les perceptions de l'identité basque et de la culture basque sur Internet. Seul le volet quantitatif, en raison de son impact, sera discuté ici. L'enquête qualitative, et les groupes de discussion en particulier, avaient une fonction de correction des résultats quantitatifs, en particulier concernant les représentations de l'identité et de la culture. Comme tout questionnaire de ce type, l'objectif de l'enquête quantitative consistait à capter les représentations individuelles autour de cinq thématiques : territoire, identité, langue, culture, pratiques culturelles. On se propose ici de revenir brièvement sur les enseignements principaux de chacune de ces rubriques.

5. A partir des cas du corse, du breton, du picard et des langues d'oïl, A. Cole et J.-B. Harguindéguy (op. cit.) distinguent trois trajectoires de l'action collective ethnolinguistique : la première consiste à matérialiser les revendications ethnolinguistiques à travers les institutions politiques et éducatives de la République ; la deuxième favorise l'usage des langues régionales en marge des institutions étatiques ; la troisième rassemble les cas de non-mobilisation. Nous situons le cas basque à l'intersection des deux premiers types. Il se distingue également par la dimension transfrontalière des mobilisations.

6. Le questionnaire a été administré par les sociétés Ikertalde côté espagnol et Stat'Adour côté français. L'INSEE Aquitaine a contribué à l'échantillonnage côté français. 
Une première salve de questions a porté sur les représentations du territoire. A la question "selon vous quel territoire définit au mieux le Pays Basque ? 7 ", les réponses ont varié en fonction de plusieurs facteurs. La représentation la plus répandue du Pays Basque comme regroupant les 7 provinces se retrouve chez ceux qui ont moins de 45 ans et chez les bascophones. Elle est plus répandue au nord du Pays Basque (35\% des enquêtés) que dans la CAB $(30 \%)$ et en Navarre (26\%), sans pour autant que l'on puisse inférer une lecture politique de cette représentation d'un territoire partagé. Les enquêtés de la CAB et de la Navarre associent le Pays Basque d'abord à la CAB, et plus précisément à la Biscaye et au Guipuzcoa, ceux du Pays Basque français au Pays Basque «intérieur » (par opposition à la Côte) ${ }^{8}$. Ce volet du questionnaire a également cherché à mesurer le sentiment de proximité entre territoires. Si ce sentiment est fort entre la CAB et la Navarre, les liens entre territoires basques du sud et du nord sont plus distendus et asymétriques. Très peu d'enquêtés de la CAB (7\%) et de la Navarre (4\%) mentionnent le Pays Basque français comme le territoire limitrophe dont ils se sentent le plus proches. La situation est inversée côté nord, où $38 \%$ des enquêtés voient le «Pays Basque sud " comme le territoire ressenti comme le plus proche, le reste de la région Aquitaine ne recueillant que $15 \%$ des réponses.

\section{Identité}

Le volet sur l'identité comportait des questions sur la hiérarchie des sentiments d'appartenance (tableau 1) et sur les composantes de l'identité basque.

Tableau 1 - Sentiment d'appartenance (Baxok et al., op.cit., p. 49)

\begin{tabular}{|l|r|l|c|}
\hline $\begin{array}{l}\text { Quant au sentiment d'être Basque et Français/Espagnol, vous-mêmes vous } \\
\text { sentez-vous...? }\end{array}$ & CAB & & $\begin{array}{c}\text { PB } \\
\text { Nord }\end{array}$ \\
\hline & 40 & Basque & 11 \\
\hline Basque & 15 & Plus Basque que Français & 5 \\
\hline Plus Basque qu'Espagnol & 28 & Aussi Basque que Français & 24 \\
\hline Aussi Basque qu'Espagnol & 3 & Plus Français que Basque & 16 \\
\hline Plus Espagnol que Basque & 6 & Français & 36 \\
\hline Espagnol & 5 & Autre & 7 \\
\hline Autre & 4 & Nsp/nrp & 1 \\
\hline Nsp/nrp & 100 & \% verticaux & 100 \\
\hline$\%$ verticaux & 46 & Double identité* & 45 \\
\hline Double identité* & & & \\
\hline
\end{tabular}

* Plus Basque qu'Espagnol + aussi Basque qu'Espagnol + plus Espagnol que Basque ou plus Basque que Français + aussi Basque que Français + plus Français que Basque 
Les résultats de cette première analyse de la superposition des identités ne sont guère surprenants. $40 \%$ des habitants de la $\mathrm{CAB}$ se considèrent comme « uniquement Basques » et $15 \%$ «plus Basques qu'Espagnols »; $28 \%$ se sentent « aussi Basques qu'Espagnols », $6 \%$ se considèrent «Espagnols » et $3 \%$ "plus Espagnols que Basques ». En Pays Basque français, ceux qui se sentent uniquement Français arrivent en tête des enquêtés (36\%). Mais ce sont surtout les doubles affiliations identitaires qui méritent d'être soulignées : $46 \%$ dans la $\mathrm{CAB}$ et $45 \%$ en Pays Basque français. En Navarre, le questionnaire a été adapté afin d'intégrer l'affirmation identitaire navarraise, aux côtés des items «Basque » et «Espagnol ». De fait, $38 \%$ des enquêtés en Navarre se sentent « d'abord Navarrais », $19 \%$ « Navarrais et Espagnol », $15 \%$ « Basque et Navarrais », $10 \%$ «d'abord Basque », $5 \%$ « d'abord Espagnol », $0 \%$ " autant Basque qu'Espagnol » et $13 \%$ « autre ». Cette dernière proportion est particulièrement significative, elle aurait mérité de plus amples développements quant aux identités référées (européenne ? issue de l'immigration ? autres ?).

Le questionnaire se penche ensuite (tableau 2, page suivante) sur les différentes composantes de l'identité basque. Est alors demandé aux enquêtés d'isoler les deux conditions les plus importantes pour qu'une personne puisse se considérer comme « Basque».

L'item «vouloir être Basque » arrive en tête, avec $42 \%$ de réponses sur l'ensemble des enquêtés. Cette réponse, renvoyant implicitement à une approche libérale et volontariste de l'appartenance, est suivie de très près $(41 \%)$ d'un critère territorial : est Basque celui qui «vit et travaille en Pays Basque » et d'un critère d'origine territoriale ("être né au Pays Basque »). Le critère linguistique (" parler basque ») arrive en retrait (17\% des répondants). Les critères militant (« être défenseur du Pays Basque »: $16 \%$ ) et essentialistes («avoir des ancêtres basques »: $9 \%$, «avoir un nom basque »: $4 \%$ ) sont moins invoqués. Ces écarts renvoient en creux à des conceptions différentes de l'appartenance : selon le critère privilégié, on peut devenir Basque, selon tel autre, le déterminisme des origines empêche toute intégration. La déclinaison territoriale des réponses est instructive : dans la $\mathrm{CAB}$, le critère volontariste ("vouloir être Basque ») arrive en tête; alors que la réponse « être né(e) au Pays Basque » l'emporte en Navarre et, de façon plus nette, en Pays Basque français. De même, la part de la langue dans la définition collective est-elle plus prononcée en Pays Basque français (40\%) que dans la CAB (16 \%) et en Navarre (10\%). Il n'y a pas de différence sensible entre hommes et femmes dans la ventilation des réponses, ni entre classes d'âge. Enfin, il est à souligner

7. Baxok et al., op. cit., p. 33 .

8. La catégorie " autres» (territoires) aurait pu être affinée en incluant un item « Navarre uniquement ", qui aurait permis de capter la position navarriste voyant dans cette province l'essence historique des provinces basques, position qui ressort des groupes de discussion navarrais. 
Tableau 2 - Représentations des composantes de l'identité basque (Baxok et al., op.cit., p. 53) 9

\begin{tabular}{|c|c|c|c|c|c|c|c|c|c|}
\hline & \multirow{2}{*}{ Total } & \multicolumn{3}{|c|}{ Territoire } & \multicolumn{2}{|c|}{ Sexe } & \multicolumn{3}{|c|}{$\begin{array}{c}\text { Connaissance } \\
\text { du basque }\end{array}$} \\
\hline & & $\mathrm{CAB}$ & Navarre & $\begin{array}{c}\text { PB } \\
\text { nord }\end{array}$ & $\mathrm{H}$ & $F$ & Non & $\begin{array}{l}\text { Un } \\
\text { peu }\end{array}$ & Oui \\
\hline $\begin{array}{l}\text { Vouloir } \\
\text { être } \\
\text { Basque }\end{array}$ & 42 & 46 & 41 & 14 & 42 & 42 & 35 & 46 & 51 \\
\hline $\begin{array}{l}\text { Vivre et } \\
\text { travailler } \\
\text { au Pays } \\
\text { Basque }\end{array}$ & 41 & 46 & 31 & 24 & 41 & 41 & 43 & 45 & 35 \\
\hline $\begin{array}{l}\text { Être né au } \\
\text { Pays } \\
\text { Basque }\end{array}$ & 39 & 36 & 42 & 53 & 38 & 39 & 44 & 37 & 30 \\
\hline $\begin{array}{l}\text { Parler } \\
\text { basque }\end{array}$ & 17 & 16 & 10 & 40 & 17 & 17 & 11 & 8 & 34 \\
\hline $\begin{array}{l}\text { Être } \\
\text { défenseur } \\
\text { du Pays } \\
\text { Basque }\end{array}$ & 16 & 17 & 13 & 13 & 16 & 15 & 14 & 16 & 18 \\
\hline $\begin{array}{l}\text { Avoir des } \\
\text { ancêtres } \\
\text { basques }\end{array}$ & 9 & 5 & 16 & 31 & 9 & 9 & 12 & 7 & 5 \\
\hline $\begin{array}{l}\text { Avoir un } \\
\text { nom } \\
\text { basque }\end{array}$ & 4 & 3 & 7 & 6 & 4 & 4 & 5 & 4 & 2 \\
\hline $\begin{array}{l}\text { Pratiquer } \\
\text { des arts } \\
\text { basques } \\
\text { (danse, } \\
\text { musique } \\
\text {...) }\end{array}$ & 1 & 1 & 1 & 2 & 1 & 1 & 1 & 1 & 0 \\
\hline $\begin{array}{l}\text { Pratiquer } \\
\text { ou suivre } \\
\text { un sport } \\
\text { ou un jeu } \\
\text { basque }\end{array}$ & 1 & 1 & 1 & 1 & 1 & 1 & 1 & 1 & 0 \\
\hline $\begin{array}{l}\text { Autre } \\
\text { condition }\end{array}$ & 3 & 3 & 5 & 3 & 4 & 2 & 2 & 6 & 3 \\
\hline Nsp/nrp & 4 & 4 & 8 & 3 & 4 & 5 & 6 & 3 & 1 \\
\hline
\end{tabular}

9. La distinction par classe d'âge, ne présentant pas d'écart significatif, n'est pas reprise ici. 
que les représentations des composantes de l'identité diffèrent sensiblement en fonction du degré de connaissance de la langue basque : si l'item volontariste ("vouloir être Basque ») arrive en tête chez les bascophones, c'est le critère d'origine ("être né au Pays Basque ») qui l'emporte chez les non bascophones. En toute logique, le critère linguistique est survalorisé par les bascophones, ainsi que le critère militant (défendre le Pays Basque), alors que les non bascophones accorderont davantage d'importance à des critères territoriaux (vivre et travailler au Pays Basque) et d'origine (lieu de naissance, ancêtres, nom) ${ }^{10}$.

\section{Langue}

Le volet proprement linguistique de l'enquête a repris les items élémentaires sur les aptitudes linguistiques (compréhension, expression orale, lecture, écriture) et sur les représentations liées à la langue, sans pour autant les développer autant que ne le font les enquêtes sociolinguistiques. Les données sur la compréhension de la langue confirment ce que les enquêtes sociolinguistiques avaient mis à jour : les niveaux les plus élevés de compréhension du basque se trouvent dans l'ordre dans la CAB, en Pays Basque français et en Navarre. Il y a une corrélation entre les niveaux élevés de compréhension de la langue et le sentiment identitaire "prédominance basque ». En ce qui concerne l'expression orale (tableau 3, page suivante), $29 \%$ des enquêtés déclarent parler «bien » ou « assez bien » le basque, la CAB arrivant en tête avec plus d'un tiers de bilingues actifs, suivie du Pays Basque français et de la Navarre. Il est à noter que le taux le plus élevé de ceux qui déclarent parler «assez bien » et «bien » le basque se trouve dans la classe d'âge des 16-29 ans, ce qui laisse augurer d'une reprise générationnelle déjà en marche depuis plusieurs années dans la $\mathrm{CAB}$ et en Navarre. Concernant le Pays Basque français, l'enquête sociolinguistique de 2006 signalera en effet que si le nombre de bilingues actifs augmente en fonction de l'âge (les taux les plus élevés se situent chez les plus de 65 ans), le nombre de bilingues est plus élevé chez les 16-24 ans que chez les 25-34 ans, ce qui constitue une inversion historique des courbes générationnelles ${ }^{11}$.

10. Le questionnaire ayant été administré en basque aux locuteurs bascophones, il a fallu adapter ce volet. "Basque » se traduit de façon distincte selon le contexte d'énonciation. Euskera désigne la langue basque. Euskaldun désigne le Basque en tant que personne : étymologiquement, est Basque la personne qui parle basque (euskara-dun). Or, ce volet de l'enquête cherchant à mesurer l'identité basque en la distinguant de l'identité linguistique, l'expression euskal herritar (du pays/peuple basque, le basque herri ayant une polysémie proche du pueblo espagnol) a été préférée à celle d'euskaldun.

11. Baztarrika Galparsoro P., Osa Ibarloza E., Iriart J.-C., Aizpurua Espin J., IV. Inkesta soziolinguistikoa 2006, Vitoria-Gasteiz : Eusko Jaurlaritza, Gobierno Vasco, 2008, p. 81. L'enquête sociolinguistique de 2006 confirme que le nombre de bilingues actifs est le plus important dans la zone Basse-Navarre-Soule, suivie du Labourd intérieur puis de l'agglomération Bayonne-Anglet-Biarritz. 
Tableau 3 - Aptitude à parler le basque (Baxok et al. op.cit., p. 59)

\begin{tabular}{|l|c|c|c|r|r|r|r|r|}
\cline { 3 - 9 } \multicolumn{1}{c|}{} & \multirow{2}{*}{ Total } & \multicolumn{4}{c|}{ Territoire } & \multicolumn{4}{c|}{ Groupes d'âge } \\
\cline { 3 - 9 } \multicolumn{1}{c|}{} & CAB & Navarre & $\begin{array}{c}\text { PB } \\
\text { nord }\end{array}$ & $16-29$ & $30-45$ & $46-64$ & $>65$ \\
\hline $\begin{array}{l}\text { Rien+ } \\
\text { quelques } \\
\text { mots }\end{array}$ & $\mathbf{5 3}$ & 44 & 77 & 65 & 33 & 48 & 62 & 69 \\
\hline Un peu & $\mathbf{1 8}$ & 22 & 10 & 8 & 25 & 22 & 15 & 9 \\
\hline $\begin{array}{l}\text { Assez } \\
\text { bien } \\
\text { bien }\end{array}$ & $\mathbf{2 9}$ & 34 & 13 & 27 & 42 & 29 & 24 & 23 \\
\hline $\begin{array}{l}\% \\
\text { verticaux }\end{array}$ & 100 & 100 & 100 & 100 & 100 & 100 & 100 & 100 \\
\hline
\end{tabular}

L'enquête de 2004-2005 met également à jour (figure 1) une corrélation entre le sentiment identitaire "prédominance basque » et la compétence (parlée) en langue basque, soit pour l'ensemble des trois territoires enquêtés :

Figure 1 - Sentiment identitaire et compétence linguistique

(Baxok et al. op.cit., p. 59)

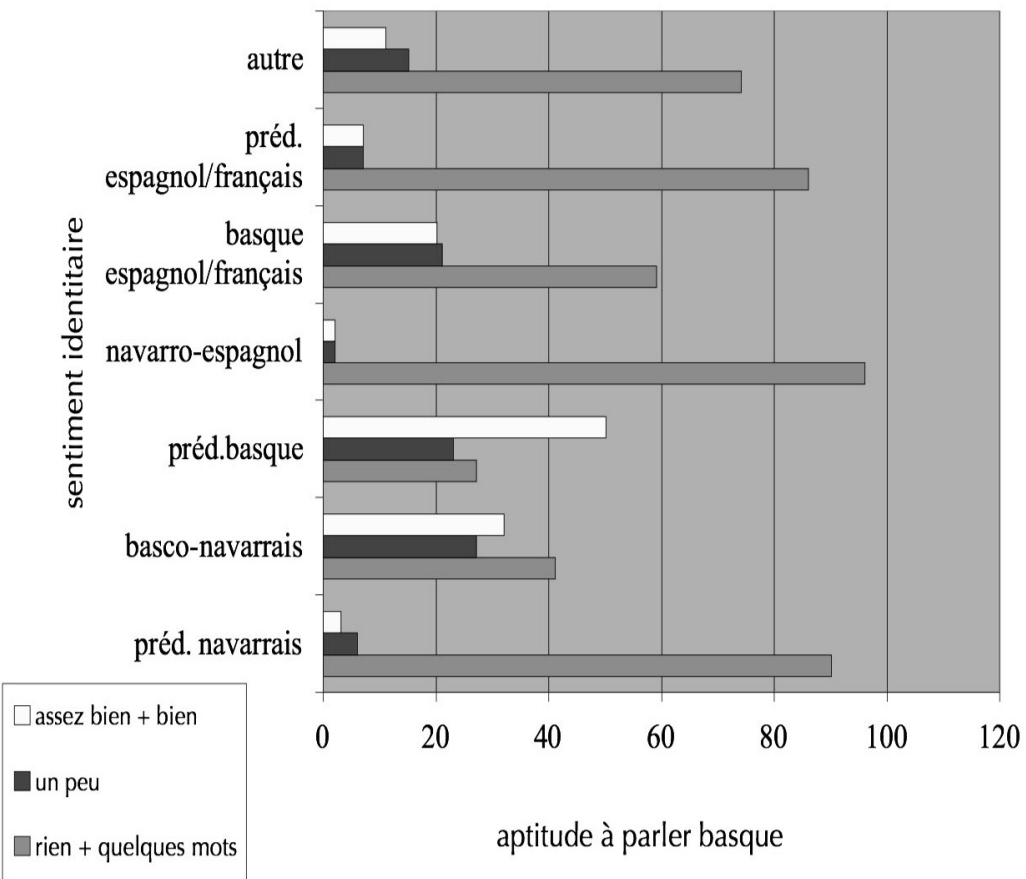


L'enquête a également cherché à mesurer, sur le plan des représentations cette fois, le sentiment d'exclusion linguistique, dans un sens comme dans l'autre. $25 \%$ des bascophones se sont déjà sentis écartés pour avoir voulu parler en basque, alors que $20 \%$ des non-bascophones ont ressenti le même sentiment pour n'avoir pas su parler basque. Paradoxalement, le taux le plus élevé de stigmatisation ressentie par les non bascophones (34\%) se trouve côté français, soit sur un territoire où l'absence d'officialité de la langue rend pourtant peu probables les situations de discrimination sociale et professionnelle pour non connaissance du basque. Peut-être ce sentiment d'exclusion est-il à relier à une moindre accoutumance, dans un contexte français, au bilinguisme, mais l'enquête ne permet pas d'approfondir cette hypothèse. Toujours sur le plan des représentations, le désir que les enfants apprennent le basque est généralisé, surtout dans la CAB. En Navarre et au Pays Basque français, ce désir est très répandu chez les bascophones et chez ceux qui se sentent basques. Chez les autres enquêtés, l'indifférence est de mise. Parmi les personnes ayant essayé ou envisageant d'apprendre le basque, deux raisons principales sont invoquées : parce que c'est la langue du pays et pour pouvoir communiquer avec les bascophones. Ceux qui n'ont aucune intention de l'apprendre soulignent ne pas avoir besoin du basque pour communiquer, dans la mesure où l'ensemble de la population parle soit l'espagnol, soit le français.

\section{Culture basque}

De l'identité et de la langue, l'enquête opère ensuite un glissement vers la culture. Au moins autant que les résultats proprement dit, c'est, nous y reviendrons, la construction des catégories de réponse qui informe sur les interrogations des commanditaires. A la question « qu'entendez-vous par culture basque ? ", les items suivant ont été proposés : a) toute création qui a trait au Pays Basque, b) toute création d'un artiste basque, c) toute création produite en Pays Basque, d) toute création en langue basque. Ces réponses induisent une réduction de la culture aux lettres et aux arts, soit à la culture comme objet potentiel des politiques culturelles. Les approches anthropologiques de la culture - difficilement repérables par le biais d'un tel questionnaire - sont provisoirement écartées ${ }^{12}$. Le principal résultat ayant attiré l'attention des commentateurs aura été le fait que moins de la moitié des enquêtés (47 \%) considèrent que la culture basque renvoie à toute création en langue basque. En revanche, lorsqu'il est demandé aux enquêtés : «Quels sont pour vous les aspects les plus importants qu'évoque la culture basque ? », c'est la langue qui arrive en tête, à la fois sur l'ensemble des enquêtés (53\%) et sur chacun des

12. Elles seront partiellement réintroduites plus loin (Baxok et al., op. cit., p. 80) sous le vocable de la culture populaire, et dans les groupes de discussion. Lors d'une présentation publique de l'enquête côté français, un participant s'étonnera de l'absence de la religion (et de l'Histoire) dans les items définissant la culture basque, comme si la sécularisation de l'identité basque avait constitué un a priori de la part des enquêteurs. En l'occurrence, c'est plutôt le souhait de ne pas intégrer de questions sur les opinions religieuses et politiques qui a orienté le choix des items. 
trois territoires. La formulation de la question induit des variations majeures dans les réponses. Enfin, la Navarre a, ici également, fait l'objet d'un questionnaire adapté sur la perception des liens entre «culture navarraise » et «culture basque ». Pour $42 \%$ des enquêtés, la culture navarraise fait partie de la culture basque. Ce résultat serait à nuancer à l'échelle infra-provinciale, au vu des contrastes sociolinguistiques internes propres à la Navarre ${ }^{13}$.

\section{Pratiques culturelles}

Enfin, un dernier volet de l'enquête a porté sur les pratiques culturelles, et en particulier dans leur rapport à la langue. Ce volet permet - outre qu'il souligne le niveau relativement faible de « consommation culturelle » quelle que soit la langue - de confronter la mesure de la compétence linguistique à celle de l'usage de la langue. La conclusion majeure est que la très grande majorité des activités culturelles s'effectue en français/espagnol. Les activités culturelles en langue basque sont plus pratiquées dans la CAB que sur les autres territoires, tout en restant minoritaires. Un autre indicateur mesure l'usage de la langue basque dans les médias et en matière de lecture. Les résultats y sont légèrement supérieurs. $22 \%$ des habitants des trois territoires regardent la télévision basque de façon quotidienne, un pourcentage plus élevé chez les jeunes. Un quart des enquêtés de la $C A B$ ne la regardent jamais, près de la moitié en Navarre et en Pays Basque français. Sur l'ensemble des territoires enquêtés, si la grande majorité des bascophones regarde la télévision basque, $6 \%$ des bascophones ne la regardent jamais. $15 \%$ des enquêtés écoutent la radio en basque au moins une fois par jour, et $65 \%$ n'y ont jamais recours. $26 \%$ des bascophones n'écoutent jamais la radio en basque ${ }^{14} .71 \%$ de l'ensemble des enquêtés ne lisent jamais de périodiques ou de livres en basque, ce qui correspond à peu près à la proportion de non bascophones dans la société, mais ce taux atteint également $28 \%$ chez les bascophones. À signaler cependant que l'usage quotidien de périodiques ou de livres en basque est plus conséquent chez les jeunes générations. Se donne ainsi à voir un écart entre un renouveau effectif de l'offre médiatique, culturelle et éditoriale en langue basque et des pratiques de consommation qui restent dominées par l'espagnol ou le français. En toute logique, au vu de la disproportion entre les offres culturelles dans chacune des langues, l'usage du basque est plus important pour les pratiques culturelles "traditionnelles » (bertsolaritza ${ }^{15}$, danse...) que pour les pratiques dites plus « universelles » (cinéma, théâtre, concerts...).

13. En 2006, la zone bascophone, au nord de la Navarre, comporte $60,1 \%$ de bilingues actifs, la zone mixte (Estella-Pampelune-Montagne) 8,3 \%, et la zone non bascophone au sud, 1,9\% (Baztarrika Galparsoro et al., op. cit., p. 141).

14. La proportion des Basques français qui écoutent quotidiennement la radio en basque (15\%) est légèrement supérieure à ceux qui regardent la télévision en basque quotidiennement $(13 \%)$, alors que le résultat est inverse pour la Navarre et la CAB. Sans doute ce résultat s'explique-t-il par l'activité des trois radios associatives Euskal irratiak bascophones en Pays Basque nord, qui proposent une offre de proximité très suivie.

15. Improvisation chantée. 
Le volet quantitatif de l'enquête se prêterait à des analyses de corrélation plus fines. Dans leur généralité cependant, ces quelques données soulèvent d'ores et déjà des débats méthodologiques et analytiques.

\section{Discussion : identité, territoire et institutionnalisation culturelle}

Nous proposons ici de revenir sur, d'une part, les enjeux méthodologiques soulevés par le volet de l'enquête portant sur les identités et, d'autre part, à partir du cas basque français, sur la nécessité d'une approche institutionnaliste pour contextualiser les résultats et pour saisir les tenants et les aboutissants d'une telle enquête.

\section{Méthodologie : vers une critique de l'identité comme catégorie pratique et scientifique?}

Les résultats de l'enquête doivent être relus à la lumière des réserves classiquement exprimées à l'égard des mesures quantitatives des affiliations identitaires. Ne sont mesurées ici que les représentations individuelles, qui ne disent rien des dynamiques collectives. L'enquête, par son ampleur, rend difficile toute comparaison diachronique, à l'exception du volet purement sociolinguistique, grâce aux enquêtes menées tous les 5 ans depuis 1991. Ces enquêtes comprenaient des questions sur l'appartenance identitaire ${ }^{16}$, mais ne traitaient pas en profondeur des représentations et des pratiques culturelles. Sur le plan des affiliations identitaires, l'observatoire sociopolitique de l'Université du Pays Basque Euskobarometro dispose également, pour la $\mathrm{CAB}$, d'enquêtes d'opinion périodiquement réactualisées.

C'est, en particulier, la mesure quantitative des appartenances identitaires qui fait débat ici. En l'espèce, les items privilégiés (du type : « vous sentez vous : plus Espagnol/Français que Basque, d'abord Basque, etc.) ne sont pas sans évoquer l'échelle des identifications (dite Moreno scale) classiquement utilisée dans bon nombre de travaux sur les mobilisations ethnonationalistes. Inspirée notamment des enquêtes de Juan J. Linz en Pays Basque à la fin des années 1970, l'échelle a été affinée par Luis Moreno pour l'analyse des appartenances identitaires en Catalogne et en Écosse ${ }^{17}$. Cette grille entend mesurer les identités duales en interrogeant les enquêtés sur la combinaison de leurs appartenances « ethnoterritoriales » (régionales) et « civiques étatiques » (nationales). Particulièrement adaptée à l'étude des territoires à identités multiples, une telle grille standardisée autorise la comparaison. Prise isolément, cette méthodologie présente des biais. A. M. Ruiz Jiménez ${ }^{18}$ note par exem-

16. On en trouvera un exemple d'exploitation dans : Joly L., «La cause basque et l'euskera ", Mots. Les langages du politique, $\mathrm{n}^{\circ} 74,2004$, pp. 73-89.

17. Moreno L., "Identificación dual y autonomía política : los casos de Escocia y Cataluña", Revista española de investigaciones sociológicas, ${ }^{\circ}$ 42, 1988, pp. 155-174.

18. Ruiz Jiménez A. M., "Los instrumentos de medida de las identidades en los estudios del CIS 
ple un écart significatif dans la mesure des identités espagnole et européenne entre deux enquêtes CIS et Eurobaromètre, toutes deux utilisant pourtant une échelle similaire, du type décrit précédemment. Au-delà des critiques envers une échelle à la fois unidimensionnelle, attitudinale et dichotomique, Ruiz Jiménez souligne que le moindre changement dans la formulation des questions et des items a des effets, ce qui est le propre de toute enquête quantitative. L'enquête basque fait montre d'un tel biais : l'importance de la langue dans les représentations de la culture, nous l'avons souligné, varie selon la formulation de la question. Il convient par conséquent de considérer ces résultats avec prudence et de les relire en ayant recours aux groupes de discussion et aux études qualitatives. Plus fondamentalement, selon A. M. Ruiz Jiménez, l'échelle force l'enquêté à établir une hiérarchie entre identités, ce qui ne correspond pas forcément à son vécu, et peine à mesurer l'intensité des affiliations.

L'enquête basque corrige en partie ce biais en mesurant le sentiment de proximité territoriale d'une part, et surtout en détaillant les éléments constitutifs de l'identité basque. Nous avons souligné que les items volontaristes ("vouloir être Basque ») l'emportaient dans la Communauté autonome basque, alors que les critères d'origine («être né(e) au Pays Basque ») l'emportaient en Navarre et en Pays Basque français, et que la part de la langue dans la définition collective était sensiblement plus importante en Pays Basque français que dans les deux autres territoires. Nous laisserons de côté ici la Navarre, en raison de la problématique spécifique de ce territoire. Hubert Pérès et Mohammad-Saïd Darviche ${ }^{19}$, compilant plusieurs enquêtes menées en Pays Basque - dont celle qui fait l'objet du présent article - depuis la fin des années 1970, confirment l'évolution vers une autodéfinition plus volontariste dans la CAB. Si l'on compare CAB et Pays Basque français, pris au pied de la lettre, ces résultats pourraient être interprétés comme mettant en évidence une approche plus politisée de l'identité dans la $\mathrm{CAB}$, plus traditionnelle, voire primordialiste, en Pays Basque français. L'importance accordée à la langue côté français irait dans le même sens dans la mesure où le basque y serait davantage transmis par la famille que par les réseaux scolaires ou d'alphabétisation. D'aucuns verraient dans ces différences l'expression de stades distincts de la politisation de l'identité des deux côtés de la frontière, avec un « retard " sensible du nord sur le sud. L'exemple basque viendrait ainsi illustrer une hypothèse selon laquelle les nationalismes ethniques périphériques évolueraient, en règle générale, d'un accent mis sur les éléments primordiaux (descendance commune, « race ", langue, traditions culturelles, religion) vers une définition basée sur la territorialité et sur l'adhésion volontariste. Juan J. Linz

y el Eurobarómetro : Problemas de validez de la denominada escala Moreno", Revista española de investigaciones sociológicas, $\mathrm{n}^{\circ} 117,2007$, pp. 161-182.

19. Pérès H., Darviche M.-S., "Comparing the dynamics of identity: the cases of Spain and France", in Darviche M.-S., Genieys W. (eds.), Multinational state-building. Considering and continuing the work of Juan Linz, Montpellier, Pôle Sud, 2008, pp. 84. 
a souligné que le primordialisme peut être la source du nationalisme, mais qu'en définitive, les implications politiques du nationalisme sont incompatibles avec ce primordialisme ${ }^{20}$. Linz a eu lui-même recours au cas basque pour illustrer cette hypothèse, avec des données recueillies à la fin des années 1970 des deux côtés de la frontière. 11,7 \% des enquêtés au nord de la frontière se déclaraient alors «Basques » ou «plus Basques que Français », alors que plus de 50 \% se déclaraient «Basques » ou «plus Basques qu’Espagnols » côté sud, la faible taille de l'échantillon côté français soumettant cependant ces résultats à caution ${ }^{21}$. Une lecture rapide de ces résultats pourrait voir dans le primordialisme des Basques français la traduction d'un stade antérieur d'une politisation identitaire arrivée à maturité côté sud.

Le contraste entre les degrés de politisation de l'identité vaut toujours dans ses grandes lignes. L'analyse demanderait cependant à être affinée, et ce au moins sur quatre plans. Tout d'abord, la comparaison ne doit pas être portée implicitement par une approche évolutionniste des nationalismes, présupposant des étapes historiques conduisant à un modèle standardisé de politisation. Les modes d'affirmation politique de l'identité sont pluriels. En particulier, le nouveau mode de gouvernance territoriale qui se développe en Pays Basque français dans les années 1990, en enrôlant des acteurs de tendances politiques différentes, se laisse mal enserrer par des approches uniquement focalisées sur le succès ou l'échec des nationalismes. Deuxièmement, les représentations individuelles mesurées ici ne disent rien de la nature des mobilisations sur chacun des territoires, et ce même si, comme le suggèrent $H$. Pérès et M.-S. Darviche 22 à partir de l'exemple basque, la nature de la mobilisation politique peut avoir un effet sur l'orientation des réponses individuelles en induisant une charge politique implicite des affiliations territoriales. Troisièmement, même si ces données permettent a minima de pointer un contraste dans les représentations individuelles de l'identité ethnonationale des deux côtés de la frontière, un examen plus approfondi de ce contraste ne saurait faire l'économie d'une approche à la fois historique et institutionnaliste des formes de politisation. Sur un registre plus prudent, on rejoindra volontiers H. Pérès et M.-S. Darviche qui relisent le volet « identités » de l'enquête de 2004-2005 en y voyant avant tout l'expression des contrastes des constructions statonationales française et espagnole. Enfin, une analyse démographique plus fine permettrait de nuancer le contraste Nord-Sud. Les approches «primordialistes » de l'identité ont été relevées sur un territoire, le Pays Basque français, où 80 \% de la population réside sur la partie côtière, soit là où l'identité basque et surtout la langue basque sont en retrait. En d'autres termes, ceux-là même à qui l'on demande de définir ce que signifie « être Basque » ne parlent pas forcément d'eux-mêmes. Il est également dommagea-

20. Linz J. J., "From primordialism to nationalism”, in Tiryakian E. A., Rogowski R. (eds.). New nationalisms of the developed West, Boston, London, Sydney, Allen \& Unwin, 1985, p. 205.

21. Linz J. J., Conflicto en Euskadi, Madrid, Espasa Calpe, 1986, p. 77.

22. Pérès H., Darviche M.-S., "Comparing the dynamics of identity...”, op. cit., p. 79. 
ble qu'aucun volet de l'enquête n'ait pas cherché à disséquer de façon analogue les composantes des identités française, espagnole et autres.

Les réserves méthodologiques soulèvent des questions plus fondamentales qui vont au-delà de la seule interrogation sur l'analyse quantitative. Ce que révèlent, en creux, les doutes exprimés ça et là lors de la réception de l'enquête mais également la perplexité de bon nombre d'enquêtés, c'est un questionnement sur le recours même au vocable d'identité. L'enquête a postulé, dans la construction de ses items, une double pertinence de l'identité en tant que catégorie pratique (pour les enquêtés) et scientifique (pour le traitement des résultats). Or, pour ne parler que de l'usage académique du concept, Rogers Brubaker ${ }^{23}$ a souligné les problèmes associés à l'usage d'un terme aussi ambigu, et a proposé d'écarter le terme au profit de l'analyse de la «communalité », de la «connexité » et de la "groupalité ». Reprenant cette analyse, Martina Avanza et Gilles Laferté 24 montrent que si le rejet des postures essentialistes de l'identité constitue désormais un acquis généralisé en sciences sociales, il est temps de soumettre à leur tour à la critique les approches constructivistes par trop radicales de l'identité. Ces dernières soulèvent en effet à leur tour trois difficultés : une posture dénonciatrice aux conséquences politiques conduisant à aborder les identités comme des «impostures », donc comme des objets sociaux indésirables ; un obstacle épistémologique plaçant prioritairement le regard du côté de la production des discours de constitution des identités au détriment de leur réception ; un désenchantement relativiste peu soucieux de l'institutionnalisation du social. Pour arriver à dépasser l'analyse du discours, il faut une compréhension institutionnaliste du monde social qui tienne compte du poids des institutions politiques, de la structure socioéconomique, de l'autorité sociale des individus ou des groupes à l'origine des discours identitaires. M. Avanza et G. Laferté jugent ainsi préférable de réserver le terme d'identité à une catégorie de la pratique et de l'abandonner comme catégorie scientifique. Ils proposent de lui substituer trois concepts alternatifs : l'identification, réservée à une sociologie du traitement bureaucratique, et qui « pourrait qualifier toute action sociale où l'attribution identitaire est extérieure, s'exerçant sur un individu, dans le cadre d'une institution sociale, selon une technique codifiée 25 » ; l'image, soit l'étude de la production sociale des discours, de symboles figurant les groupes et les territoires; l'appartenance ou comment les identifiés ou représentés s'approprient, refusent, acceptent ces identifications et ces images. Les auteurs plaident ainsi en faveur d'un constructivisme institutionnel et structurel permettant de bien saisir les processus à l'œuvre derrière les pratiques et les références identitaires.

23. Brubaker R., «Au-delà de l'identité », Actes de la recherche en sciences sociales, $\mathrm{n}^{\circ} 139,2001$, pp. 66-85.

24. Avanza M., Laferté G., «Dépasser la “construction des identités” ? Identification, image sociale, appartenance », Genèses, 61, décembre 2005, pp. 134-152.

25. Ibid., p. 142. 
En accord avec cette dernière approche, il nous semble que les résultats de l'enquête de 2004-2005 ne prennent leur dimension véritable qu'à la lumière d'une analyse serrée des évolutions institutionnelles des territoires. A ce titre, l'observation de l'institutionnalisation territoriale du Pays Basque français, si elle ne permet pas d'expliquer les permanences des contrastes entre représentations de l'identité et de la culture des deux côtés de la frontière, permet du moins d'élucider les conditions de production et de réception de l'enquête. En l'occurrence, la nature même des opérateurs de l'enquête informe sur l'asymétrie institutionnelle entre les trois territoires convoqués : un organisme public pour la $\mathrm{CAB}$ (le service de prospection sociologique du Gouvernement basque), une association transfrontalière d'études basques (Eusko ikaskuntza) pour l'enquête navarraise et la publication de l'enquête, une structure à statut associatif mais fortement liée aux institutions publiques pour le versant français (ICB).

Durant les travaux préparatoires de l'enquête, les partenaires se sont accordés en faveur de l'inclusion d'un volet « représentations de la culture » et "pratiques culturelles» dans l'enquête. Ce volet de l'enquête constitue, en particulier pour les institutions culturelles, un sondage destiné à éclaircir un questionnement sur leur propre identité institutionnelle : comment constituer la culture basque en secteur d'action publique ? Le débat est particulièrement marqué côté nord : comment constituer un secteur culturel basque qui se distingue à la fois des institutions de politique culturelle déjà existantes sur le territoire, en particulier sur la Côte urbanisée, et des institutions de politique linguistique en voie de stabilisation ? Ce double encadrement rend difficile l'émergence d'une politique culturelle aux frontières stabilisées : comment découper une réalité sociétale multiforme en un secteur d'action publique ? Qu'est-ce que la culture basque ? La culture en langue basque ? Toute production culturelle effectuée en Pays Basque ? Les incertitudes autour du statut de la langue dans la définition de la culture font débat. Des mises en garde contre le risque d'une culture basque déconnectée de sa dimension linguistique s'expriment, à l'occasion de la publication de l'enquête, de la part des réseaux militants de la langue basque. D'aucuns pointent également l'irruption des logiques d'intérêts dans un «marché culturel » basque jusque-là dominé par les logiques militantes, et aujourd'hui soumis à un double processus d'institutionnalisation et de marchandisation.

Le débat sur la culture émerge en toute logique sur un territoire qui, depuis la fin des années 1980, expérimente une transformation structurelle de l'action publique. À l'initiative d'un « sous-préfet développeur », une série de concertations entre représentants de la société civile, acteurs politiques et économiques s'articule alors autour d'une démarche prospective, «Pays Basque 
2010 ». La procédure se traduira, entre autres, par la création d'un Institut culturel basque (ICB) en 1990, d'un Conseil de développement du Pays Basque et d'un Conseil des élus du Pays Basque en 1994-1995 26. Se dessine ainsi une nouvelle forme de gouvernance territoriale largement ouverte à la société civile. La dynamique d'institutionnalisation et de sectorialisation s'adresse alors à des enjeux jusque là confondus dans l'action militante généraliste : développement économique, agriculture, culture, langue et environnement.

Dans ce contexte, le devenir de la langue et de la culture basques est passé d'un enjeu social à un problème public, et d'un problème public à un problème politique. Un problème ne devient public que lorsque des acteurs mobilisés «parviennent à l'inscrire dans l'espace public, c'est-à-dire lorsqu'il devient un sujet d'attention, voire de controverses et que des positions se confrontent pour en caractériser les composantes, l'ampleur et les causes 27 ». Tout le travail associatif depuis les années 1950 s'emploie à cette transformation ${ }^{28}$. Le problème public devient ensuite un problème politique lorsque la mobilisation parvient «à produire une visibilité suffisante et à acquérir une capacité d'interpellation telle que des acteurs qui n'étaient pas initialement concernés par l'enjeu prennent des positions et argumentent en sa faveur ou contre lui $29 »$. La puissance publique est sollicitée pour le règlement de la question. Durant les années 1990, les militants de la culture et de la langue parviennent à inciter l'ensemble des acteurs politiques territoriaux à se positionner sur l'enjeu qu'ils défendent, et ce bien au-delà des seuls secteurs nationalistes. Enfin, la question linguistique et culturelle parvient à entrer dans l'agenda gouvernemental local, bénéficiant notamment d'une attitude globalement plus favorable de la part de l'État. La mise sur l'agenda politique et l'institutionnalisation qui en a résulté s'opèrent d'abord pour le volet culturel. Celle-ci ne se déroule pas sans heurts, puisque la création de l'ICB ne se fera en 1989-1990 qu'au terme d'une séparation d'avec les institutions culturelles "généralistes » de la Côte basque ${ }^{30}$. La promotion de la langue basque, dans cette première phase, s'effectue par l'ICB d'une part, par les réseaux scolaires (immersif associatif, bilingue public et bilingue catholique ${ }^{31}$ ) et d'alphabétisa-

26. Chaussier, J.-D., Quel territoire pour le Pays Basque ? Les cartes de l'identité, Paris, L'Harmattan, 1996.

27. Lascoumes P., Le Galès P., op. cit., p. 69.

28. Oronos M., Le monvement culturel basque (1951-2001). 1. Ikas, Pizkundea, Euskal Konfederazioa, Bayonne, Elkar, 2002, Bortayrou J., Etcheverry-Ainchart P., Garat M., Leralu C., Lichau I., Le mouvement culturel basque (1951-2001). 2 : Filières bilingues, Seaska, $A E K$, Bayonne, Elkar, 2005.

29. Lascoumes P., Le Galès P., op. cit., p. 80.

30. En 1984, le Centre Culturel du Pays Basque est créé. L'institution diffuse, crée et coproduit des spectacles. En 1988, ce Centre est dissout puis réapparaît sous la forme du Centre d'Action Culturelle de Bayonne et du Sud-Aquitaine d'une part, et de l'ICB d'autre part (Laborde D., " Politique culturelle et langue basque ", in Blanchet P., Breton R., Schiffman H. (dir.), The Regional Languages of France : an Inventory on the Eve of the 21st Century, Louvain-la-Neuve, Peeters, 1999, pp. 141-160).

31. Les filières d'enseignement du basque ont leur propre dynamique d'institutionnalisation, avec la signature de contrats d'association avec l'État à compter du début des années 1990. 
tion d'autre part. Rapidement cependant, le besoin d'une structure spécifique pour la promotion d'une politique linguistique autonome, que l'on entend désormais distinguer de l'action culturelle proprement dite, se fait ressentir. Au terme d'un long processus, l'institutionnalisation d'une politique linguistique favorable à la langue basque voit progressivement le jour : la langue basque s'inscrit pour la première fois au cœur d'un projet territorial avec le Schéma d'aménagement du Pays Basque en 1996-1997. Cette structuration est renforcée par la Convention Spécifique Pays Basque en 1999-2000. Les institutions se réunissent alors autour d'un même programme d'aides aux opérateurs linguistiques (2000-2004) et se coordonnent au sein d'une maitrise d'ouvrage public, le Conseil de la langue basque. En janvier 2005, l'Office public de la langue basque (OPLB), à statut de groupement d'intérêt public, est créé. Il associe représentants de l'État (préfecture, rectorat, Direction Régionale des Affaires Culturelles), de la Région Aquitaine, du Département des PyrénéesAtlantiques, du Syndicat intercommunal de soutien à la culture basque et du Conseil des élus du Pays Basque. Ses missions consistent à aider les opérateurs de l'action linguistique, à coordonner l'offre et la demande d'enseignement du basque et en basque sur le territoire, et à assurer une promotion de la langue dans la vie sociale et auprès des pouvoirs publics ${ }^{32}$.

La dissociation entre politique linguistique et politique culturelle, déjà expérimentée en Bretagne, soulève un débat inédit en Pays Basque. Si le découpage des secteurs d'intervention de l'OPLB est relativement clair, il renvoie en miroir aux incertitudes sur le devenir de l'ICB qui se voit, pour le coup, forcé de repenser son positionnement à un double niveau. Sur le plan légal, d'une part, les discussions portent désormais sur le maintien du statut associatif ou le passage à un statut public (type établissement public de coopération culturelle) pour une structure fortement soutenue par les collectivités territoriales et l'État. Plus fondamentalement, ce sont les frontières de son domaine d'action (patrimoine, spectacle vivant, promotion des artistes, soutien aux pratiques « traditionnelles ", etc.) qui animent les discussions au sein de l'ICB. La dynamique d'institutionnalisation culturelle devient, de fait, complexe sur un territoire où les dynamiques culturelles sont déjà fortement structurées soit par un mouvement social basque transfrontalier ${ }^{33}$, soit par des dispositifs coutumiers et associatifs à l'échelle de la vallée ou du village et reposant sur les classes d'âge, le voisinage, les groupes affinitaires, la paroisse, etc.

Cette profusion d'initiatives et de dynamiques n'est d'ailleurs pas forcément préjudiciable à l'action publique. Vincent Dubois a montré comment, à l'échelle française, les politiques culturelles reposaient précisément sur la

32. Entre autres dispositifs, l'OPLB propose aux communautés de communes et aux communes de grande taille des «techniciens de la langue » pour le soutien à la promotion locale du basque.

33. Letamendia F. (eds), Acción colectiva Hegoalde-Iparralde, Madrid, Fundamentos, 2006. 
construction de la culture comme une catégorie floue d'action publique : "L'indécision de la politique culturelle, la remise périodique sur le métier de sa définition constituent moins les signes d'une "faiblesse", d'un "manque", que la voie particulière suivie par son institutionnalisation 34 ». Lorsqu'à ce flou intrinsèque, propre au champ culturel, se greffe la problématique identitaire, toutes les conditions sont réunies pour que l'entreprise d'institutionnalisation de la culture basque se heurte à des difficultés récurrentes. Or, le flou autour de la culture basque - comme de la culture tout court - loin de constituer un obstacle, constitue également une ressource pour des institutions (l'ICB et les autres opérateurs culturels) en quête de stabilité au sein d'un paysage culturel aussi fragmenté que dynamique. Tous s'accordent, enfin, pour reconnaître aux nouvelles institutions de la gouvernance culturelle du territoire une fonction de médiation entre des milieux institutionnels, associatifs et militants, aux relations jusque-là fortement teintées de défiance. S'institutionnalise dès lors une médiation culturelle basque soumise à la vigilance des milieux militants mais également confrontée à la réactivation, dans les années 2000 , d'un militantisme associatif républicain et centraliste opposée à toute co-officialisation de la langue basque ${ }^{35}$.

Ce n'est qu'à l'aide de ces évolutions territoriales de fond que doivent se lire les résultats d'une enquête dont la relecture critique n'entend pas minorer l'utilité. Bien au contraire. Par sa dimension, par les tendances et par la pluralité des appartenances qu'elle donne à voir, l'enquête fera date, à condition de la compléter par des études qualitatives qui ne fassent pas l'économie d'une analyse néo-institutionnaliste. Les institutions sont influencées par les perceptions individuelles, mais en retour les dynamiques d'institutionnalisation de la langue et de la culture basque ont influé sur les perceptions individuelles et notamment sur la diffusion normalisée de la promotion publique de la langue basque. En Pays Basque français, l'institutionnalisation contribue d'ores et déjà à inverser le stigmate social longtemps associé à la langue. Les prochaines études sociolinguistiques diront si cette politique volontariste se traduit par une inversion des courbes en termes de compétence - une inversion déjà observée en 2006 - et surtout d'usage de la langue. En matière culturelle, le pluralisme des représentations de la culture basque, telles qu'elles ont été saisies par l'enquête de 2005, répond, en écho, au questionnement à propos d'une institutionnalisation culturelle en devenir. L'ensemble de ces incertitudes, qu'elles soient scientifiques ou pratiques, témoigne des dynamiques plurielles d'un territoire expérimentant une transformation sociopolitique profonde, inédite et aux effets durables.

34. Dubois V., « Politique culturelle : le succès d'une catégorie floue. Contribution à une analyse des catégories d'intervention publique », in Kaluszynski M., Wahnich S. (dir.), L'État contre la politique? Les expressions politiques de l'étatisation, Paris, L'Harmattan, 1998, p. 168.

35. Voir Pierre T., Controverses institutionnelles en Pays Basque de France. Usages politiques et déconstructions des préjugés socioculturels, Paris, L'Harmattan, 2010, chap. 5 et 9. 